\title{
Regulation of the serotonin neuron fate in stem cells by foxa2 and shh
}

\begin{abstract}
Serotonin neurotransmission plays an important role in controlling mood, cognition, and various physiological functions. The development of serotonin neurons in the embryo, however, is not well understood. Serotonin neurons are born on the ventral side of the fetal hindbrain. Prior reports suggested that mechanisms responsible for the induction of dopamine neurons in the midbrain are also responsible for the generation of serotonin neurons in the hindbrain. We have previously shown that dopamine neurons are derivatives of the medial floor plate of the midbrain and that the floor plate-specific transcription factor, foxa2, is necessary and sufficient for the development and differentiation of dopamine neurons. Here, we have examined the development of serotonin neurons with respect to the hindbrain floor plate. We show that serotonin neurons are derivatives of the lateral floor plate of the hindbrain. We further show that foxa2 is necessary and sufficient for the development of serotonin neurons. Finally, we show that increased shh signaling can induce serotonin neurons in midbrain, as well. These results demonstrate that while the organization and potential of the floor plate differs between the hindbrain and midbrain, progenitors of serotonin neurons, like those of dopamine neurons, are also critically regulated by foxa2.
\end{abstract}

Keywords: stem cells, serotonin neurotransmission, fgf8, shh, fgf4, $\operatorname{lmx} 1 \mathrm{~b}$, maoa
Volume 3 Issue 2 - 2017

\author{
Raja Kittappa, ${ }^{1,2}$ James A Kehler, ${ }^{3}$ Christina S \\ Barr' \\ 'Laboratory of Neurogenetics, Section of Comparative \\ Behavioral Genomics, National Institutes of Health, USA \\ ${ }^{2}$ Navastra Inc., USA \\ ${ }^{3}$ Laboratory of Cell and Molecular Biology, National Institute of \\ Diabetes and Digestive and Kidney Diseases, National Institutes \\ of Health, USA
}

Correspondence: Raja Kittappa, Laboratory of Neurogenetics, Section of Comparative Behavioral Genomics, National Institute of Alcoholism and Alcohol Abuse, National Institutes of Health, Bethesda, MD 20892, USA, Tel 30|4968।27.

Email raja.kittappa@gmail.com

Christina S Barr, Laboratory of Neurogenetics, Section of Comparative Behavioral Genomics, National Institute of Alcoholism and Alcohol Abuse, National Institutes of Health, Bethesda, MD 20892, USA, Tel 30|4968|27, Email cbarr@mail.nih.gov

Received: June 08, 2017 | Published: July 21, 2017

\section{Introduction}

The central serotonergic system is comprised of tryptophan hydroxylase-expressing neurons located in the raphe nuclei of the brain stem. Rostral groups of raphe serotonin neurons project throughout the forebrain, while caudal groups innervate the brain stem and spinal cord. ${ }^{1}$ The serotonergic system modulates mood, ${ }^{2}$ anxiety, ${ }^{3}$ aggression, ${ }^{4}$ reward systems, ${ }^{5}$ and impulsivity, ${ }^{6}$ and psychosis. ${ }^{7}$ Many psychiatric diseases, such as depression, obsessivecompulsive disorder, and bulimia nervosa are currently treated by medications targeting the serotonergic system. ${ }^{8,9}$ Abnormalities in the serotonergic system have also been implicated in diseases affecting early development such as sudden infant death syndrome ${ }^{10}$ and autism. ${ }^{11}$ Recent evidence suggests that dysregulation of serotonin neuron development may underlie behavioral changes. The activity of 5HT1A receptor during postnatal development has been shown to be important for normal anxiety behavior in adult mice. ${ }^{12}$ The loss of Pet-1, a transcription factor important for the development of serotonin neurons in the embryo has been shown to cause increased anxiety and aggression in adult mice. ${ }^{13} \mathrm{~A}$ more precise understanding of the development of the serotonergic system may be critical for understanding the relationship between early changes in serotonergic regulation and the pathophysiology of psychiatric disorders, autism, and sudden infant death syndrome.

Serotonin neurons are first detected in the ventral hindbrain between E11.5 and E13.5 in the mouse embryo and between five and six weeks of gestation in the human fetus. ${ }^{14,15}$ Previous studies have suggested that the serotonergic fate is induced by the combinatorial actions of a signal from the rostrally situated midbrain-hindbrain boundary, the isthmus; a ventralizing signal from the ventral midline organizer of the neural tube, the floor plate; and, a posteriorizing signal whose source has yet to be precisely defined. ${ }^{16}$ Candidates for the signals inducing serotonergic fate from the isthmus and floor plate and, also, the posteriorizing signal have been proposed to be fgf8, shh, and fgf 4 , respectively. ${ }^{17}$ In addition, these molecules have been shown to promote the differentiation of serotonin neurons from embryonic stem cells. ${ }^{18}$

It is generally thought that serotonin neurons, derived from the hindbrain, are developmentally analogous to dopamine neurons, catecholaminergic neurons which arise from the midbrain. The relatedness of these cell types has been lent support, conceptually, by manipulations of the rostrocaudal position of the isthmus, using mouse genetics. When the isthmus is moved rostrally, more serotonin neurons are generated at the expense of rostral dopamine neurons; reciprocally, if the isthmus is shifted caudally, serotonin neurons are reduced while dopamine neurons expand rostrally. ${ }^{19}$ These observations suggest that serotonin neurons and dopamine neurons are developmentally related, but they do not address what common genes and pathways coordinately determine the fates of these neurons. An earlier study claims that serotonin and dopamine neurons are both induced by fgf 8 and shh with fgf 4 additionally cooperating to induce the serotonergic fate, specifically, in the hindbrain. In contrast, the absence of fgf4 signaling, in the midbrain, leads to the differentiation of dopamine neurons, there. ${ }^{17}$

Recently, we have shown that the dopaminergic fate, in the midbrain, is not induced by the floor plate; instead, the dopamine progenitors are derivatives of the floor plate itself. ${ }^{20}$ Specifically, dopamine progenitors are a medial subset of floor plate cells which is an organizer that secretes the morphogen, shh, and which was not 
previously thought to give rise to any neural cells. We also showed that the floor plate-specific forkhead transcription factor, foxa2, is both necessary and sufficient for the development of dopamine neurons. In contrast, our lineage-tracing experiment showed that serotonin neurons are not derived from the shh-expressing cells of the medial floor plate. These findings contradict earlier models of dopaminergic induction and the notion that dopaminergic and serotonergic neurons are developmentally analogous. Here, to resolve these seeming contradictions, we have investigated the relationship between the floor plate of the fetal hindbrain and serotonin progenitors and neurons. We confirm that serotonergic precursors are not located in the ventral midline and show that they are, instead, found on either side of the medial floor plate. Serotonin progenitors occupy the lateral floor plate, and express the floor plate marker, foxa2, along with the serotonin progenitor marker, nkx2.2. We show that foxa 2 is necessary and sufficient for the development of serotonin neurons in vitro. Most importantly, we further demonstrate that foxa 2 is required cell-autonomously for the differentiation of dopamine and serotonin neurons and cannot be replaced by cell non-autonomous, secreted factors from the floor plate.

\section{Results}

Mapping of progenitor domains in the spinal cord and midbrain has proven to be useful in elucidating mechanisms of fate determination and differentiation in these regions of the CNS. ${ }^{20-22}$ Initial maps of the embryonic ventral hindbrain have been generated, but these maps do not include markers of the floor plate, which has been considered non-neurogenic. ${ }^{23,24}$

Lmx $1 b$, which is expressed in the mesencephalic, medial floor plate, is also expressed in the medial floor plate of the hindbrain (Figure 1A). Lmx1b-positive nuclei can also be observed pially, but not at the ventricular surface, lateral to the medial floor plate. Consistent with previous published reports, a ventral subset of these lateral $1 \mathrm{mx} 1 \mathrm{~b}$-expressing cells are serotonin neurons (Figure 1A); however, the dorsal $1 \mathrm{mx} 1 \mathrm{~b}$-expressing cells are non-serotonergic, at least at this stage. The lateral, presumptive neurons are more intensely stained for $\operatorname{lmx} 1 b$ than the medial floor plate. In addition to the ventral staining, $1 \mathrm{mx} 1 \mathrm{~b}$-expressing cells are seen widely along the lateral midbrain (Figure 1A).

$\mathrm{Nkx} 2.2$ is a homeodomain-containing transcription factor which has been shown to be expressed in the domain immediately lateral to the floor plate in the spinal cord and the midbrain. ${ }^{20,21,25}$ Additionally, $\mathrm{nkx} 2.2$ has been shown to be expressed in progenitors of serotonin neurons and to be necessary for the development of serotonin neurons. We observe that nkx2.2 is expressed in the lateral aspect of the foxa2expressing domain but is absent from the medial part of the foxa2 domain (Figure 1B). The coexpression of $n k x 2.2$ and foxa2 in the hindbrain suggests that serotonin neurons are derivatives of the lateral floor plate. This observation is consistent with our lineage tracing experiment in which the derivatives of shh-expressing progenitors generated neurons in the midbrain but not in the hindbrain $(20 ; 28)$. Just as in the midbrain, foxa2 is downregulated in the lateral floor plate of the hindbrain, and accordingly, foxa2 is not expressed in serotonin neurons, unlike midbrain dopamine neurons which maintain foxa2 expression (Figure 1C).

Just as in the fetal midbrain, phox $2 b$-expressing motoneurons and their progenitors can be found immediately lateral to the monoaminergic neurons and their precursors ${ }^{24}$ and Supp. Figure 1A. We additionally observe a population of numerous phox $2 \mathrm{~b}$-expressing cells at a more lateral position (Supplementary Figure 1B). A sparse and discontinuous line of phox $2 \mathrm{~b}$-expressing cells, at the pial surface, connect the ventral population of motoneurons with the more lateral phox $2 \mathrm{~b}$-expressing population, at rostral levels of the hindbrain. In more caudal sections, phox $2 \mathrm{~b}$-expression is continuous at the pial surface between the presumptive motoneurons and the more lateral population (Supplementary Figure 1B). In still more caudal sections, a dorsolateral population of phox $2 \mathrm{~b}$-expressing cells is apparent. Phox2 homeoproteins are expressed in and required for the development of noradrenergic neurons in the hindbrain but the noradrenergic progenitor domain has not been defined. Both the ventrolateral and dorsolateral phox-2b-expressing cells are candidates for noradrenergic progenitors. The breadth of the phox $2 \mathrm{~b}$-expressing domain at the pial surface of the ventral hindbrain suggests that phox $2 b$ is upregulated in a large number of postmitotic neurons, shortly after differentiation. We have observed a similar effect with regard to $\lim 1 / 2$ in the developing, ventral midbrain. ${ }^{20}$

Engrailed homeodomain proteins are expressed widely during the development of the CNS and are important for the development of dopamine neurons and serotonin neurons. ${ }^{26}$ In addition, engrailed proteins have been shown to bind foxa 2 and the two proteins coordinately regulate gene expression. In this regard, we were curious to see how engrailed proteins are expressed in the hindbrain with respect to the floor plate and serotonin neurons. In more rostral sections, we observe two small clusters of cells lateral to the floor plate (Supplementary Figure 1B). These cells may contribute to the superior olive nucleus, a ventral hindbrain derivative that has been shown to express engrailed-1 at the time of birth. In more caudal sections of the hindbrain, engrailed proteins are expressed in numerous cells around the ventral midline and, at more ventrolateral locations, in a large number of presumably postmitotic cells at the pial surface (Supplementary Figure 1C). At the same rostrocaudal level, engrailed is expressed throughout the dorsal hindbrain in a gradient with maximal expression at the dorsal midline (Supplementary Figure 1C). These results suggest that, unlike foxa2, engrailed does not have a common role in the development of midbrain dopamine neurons and serotonin neurons in the hindbrain.

We present an updated schematic representing ventral hindbrain progenitor domains in the context of the floor plate, in Supplementary Figure 2. This model predicts that, unlike the midbrain, neurons do not derive from the medial floor plate of the hindbrain and, also, that serotonin neurons, from the lateral floor plate, are the ventralmost neuron in the developing hindbrain. In the E12.5 hindbrain, newly born neurons are labeled with the Tuj1 antibody which recognizes betatubulin (green, Figure 2B). While neurons are clearly observed almost to the full circumference of the hindbrain, an obvious gap in Tuj1 expression can be seen at the ventral midline, suggesting that neurons are not born here. In contrast, Tuj1 is expressed continuously across the midline and no gap is observed (Figure 2A). This is consistent with genetic lineage tracing studies performed in the hindbrain. ${ }^{20}$ The most ventral Tuj1-positive cells in the hindbrain, additionally and as predicted, are 5-HT immunoreactive.

We have recently shown that the foxa 2 forkhead transcription factor is both necessary and sufficient for the embryonic development of midbrain dopamine neurons but such a role has not been demonstrated for serotonin neurons. Foxa2 nullizygous embryos do not survive past E10.5. ${ }^{20}$ To test for a requirement for foxa2, we cultured hindbrain explants from both wild-type and foxa2 nullizygous E9.5 embryos and allowed them to differentiate. While wild-type explants readily generate hundreds of serotonin neurons, we were unable to detect 
a single serotonin neuron in foxa2 mutant explants (Figure 3A). We have previously developed methods for the differentiation of dopamine and serotonin neurons from mouse embryonic stem (mES) cells. ${ }^{18,27}$ We have also shown that the induction of foxa2 expression in a doxycycline-inducible mES line, during neuronal differentiation, significantly enhances dopaminergic differentiation. ${ }^{20}$ Here, using the same reagents, we show that the overexpression of foxa 2 in differentiating mES cells leads to a greater than 8-fold increase in the number of serotonin neurons generated (Figure 3B). Additionally, we observe that the shh inhibitor, cyclopamine, suppresses the differentiation of serotonin neurons from mES cells, just as it does to the differentiation of dopamine neurons. ${ }^{20}$ This suppression of serotonergic differentiation is similarly overcome by overexpression of foxa2 during differentiation (Figure 3C). Taken together, these results suggest that foxa 2 is both necessary and sufficient for the development of serotonin neurons.
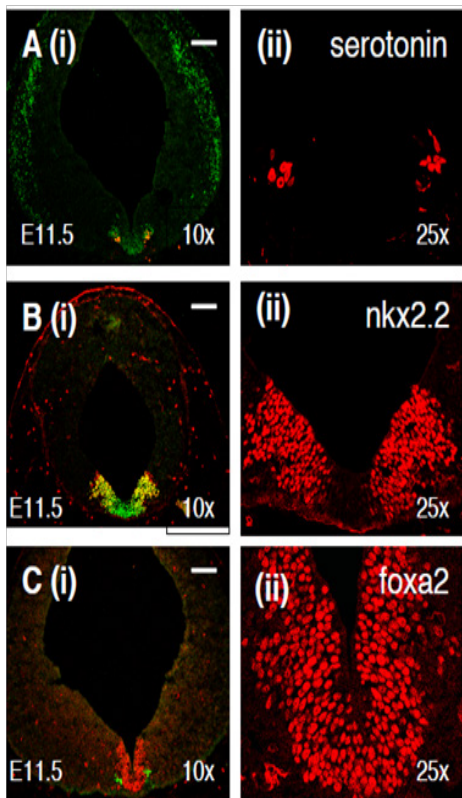
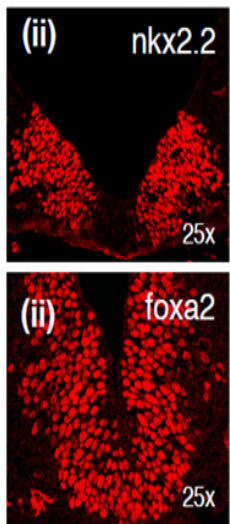
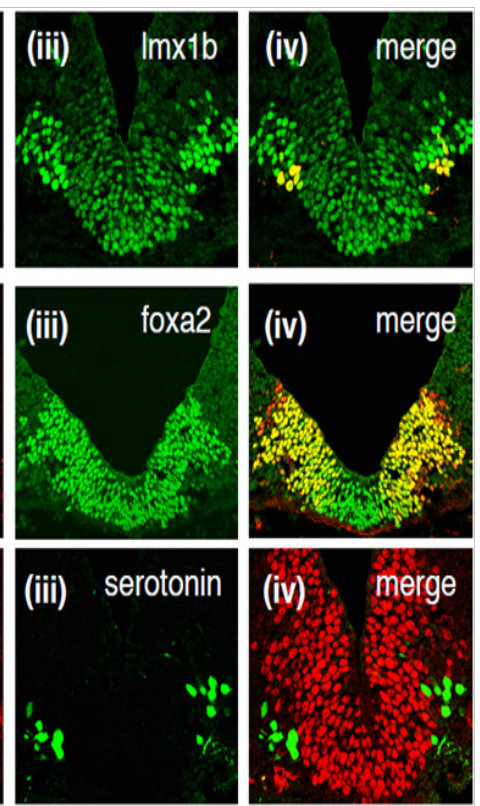

Figure I Expression of floor plate markers in relation to serotonin neuron development. (A) Lmx Ib (green) is weakly expressed at the midline in the medial floor plate and much more strongly expressed in neurons immediately adjacent to the medial floor plate. Serotonin immunoreactivity (red) is seen in the ventralmost Imxlb-expressing neurons. (B) Nkx2.2 (red) which is expressed in serotonin progenitors is coexpressed with foxa2 in (green) in the lateral parts of the foxa2-expressing floor plate. (C) While serotonin neurons (green) appear to arise from the lateral foxa2-expressing progenitors, they do not continue to express foxa2 when they differentiate. Bars in all figures signify 100 microns.
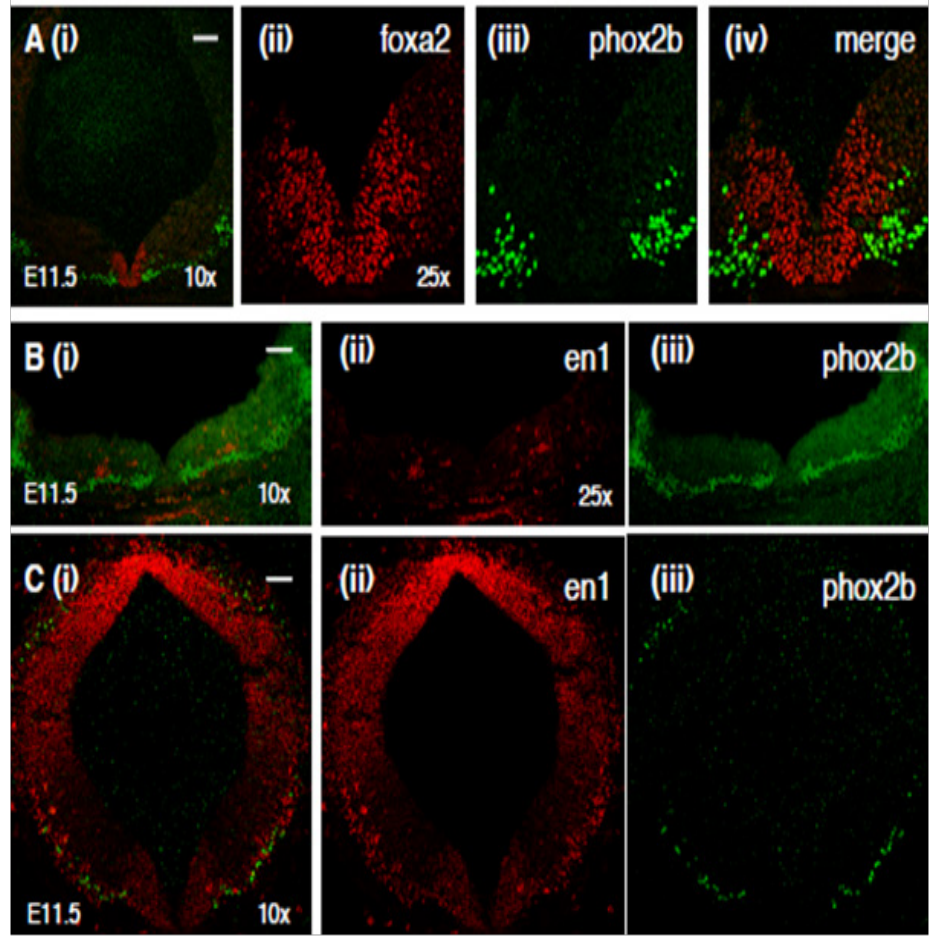

Supplementary Figure I Schematic of neurogenic domains of the ventral hindbrain. 

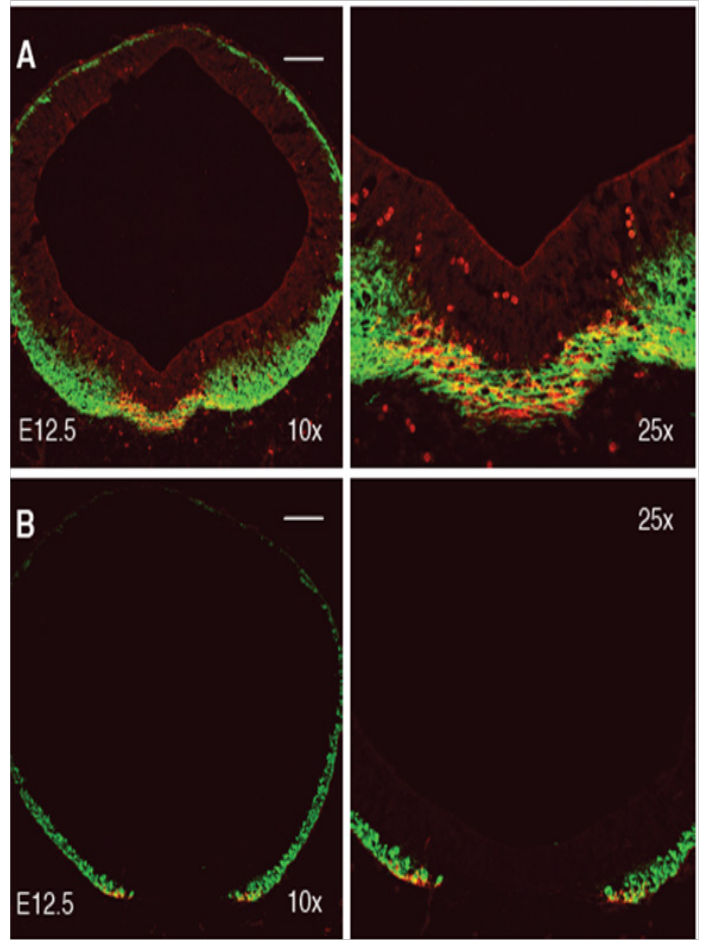

Figure 2 The floor plate is neurogenic in the midbrain but not the hindbrain. (A) Neurons, stained for beta-tubulin (Tuj I, green), are generated continuously across the ventral midline. Neurons at the ventral midline of the developing midbrain are predominantly stained for the dopamine neuron marker, tyrosine hydroxylase (TH, red). (B) In contrast to the midbrain, neurons (green) are not generated at the ventral midline of the hindbrain. Serotonin neurons (red), the ventralmost hindbrain neurons, flank the floor plate.

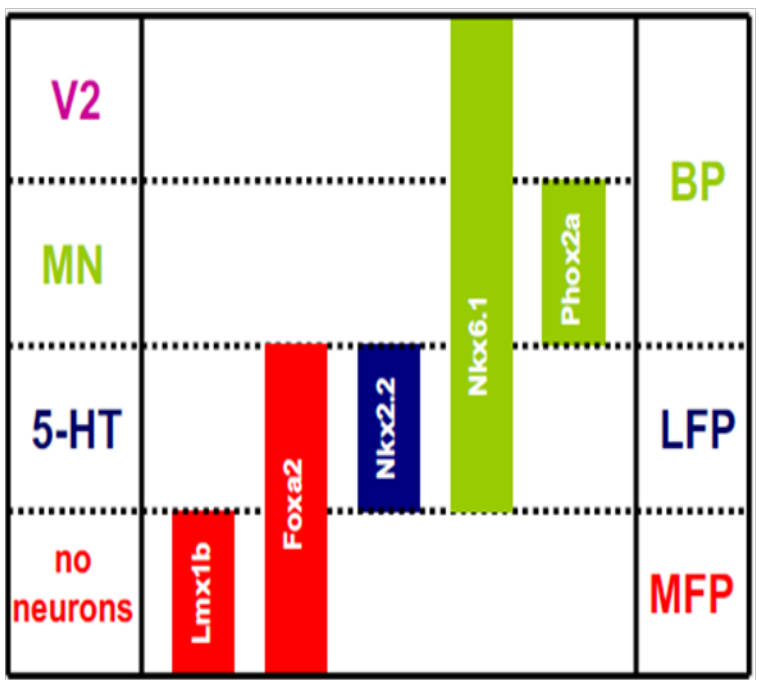

Supplementary Figure 2 Expression of homeodomain transcription factors relative to the hindbrain floor plate. (A) Phox2a--expressing motoneurons (green) are found adjacent to the foxa2-expressing floor plate (red).Additional phox $2 \mathrm{a}(2 \mathrm{~b}$ ?)-expressing cells can be seen lateral to the motoneuron domain at I0x. (B) En I (red) and phox2a (green) expression at a more caudal level than (A). Engrailed is seen in two small domains lateral to the floor plate. Phox $2 a$ is expressed in an unusually wide domain, clearly larger than the motoneuron domain. (C) At still more caudal levels, engrailed (red) is expressed strongly at the dorsal midline and in a dorsoventral gradient. Phox2a) is expressed in presumptive neurons along the breadth of the dorsoventral axis.
Many secreted factors, including shh, netrin-1, TGF-beta, wnt-1, and others, are expressed in the floor plate. This raises the possibility that the effects of foxa 2 on the differentiation of dopamine and serotonin neurons are neither direct nor cell-autonomous. The classical embryological method to study cell-autonomy is by mixing genetically distinct cells in vitro or analyzing genetically mosaic organisms. To address this issue, we co-cultured dissociated midbrain and hindbrain progenitors from H2-GFP transgenic and foxa2-/embryos of the same age. In these experiments, all of the differentiated serotonergic and dopaminergic neurons express GFP (Figure 4). The foxa2 (and GFP)-expressing progenitors, in these experiments, are unable to rescue monoaminergic differentiation, indicating that foxa2 is autonomously required to differentiate into a serotonin or a dopamine neuron.

\section{A Differentiation of Serotonin Neurons}

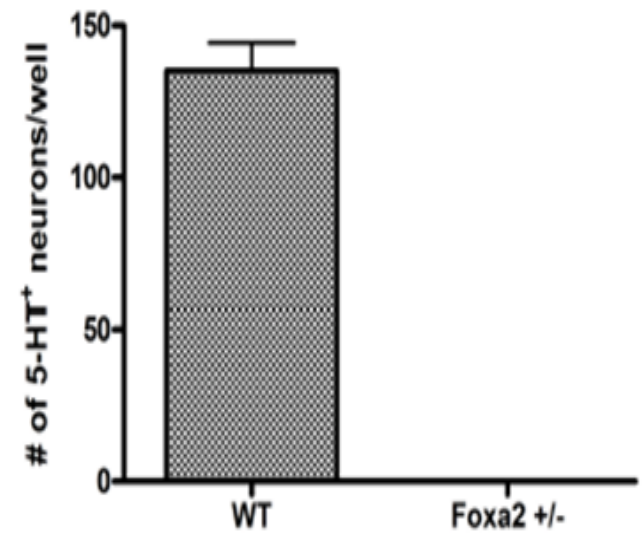

\section{B Differentiation of Serotonin Neurons}

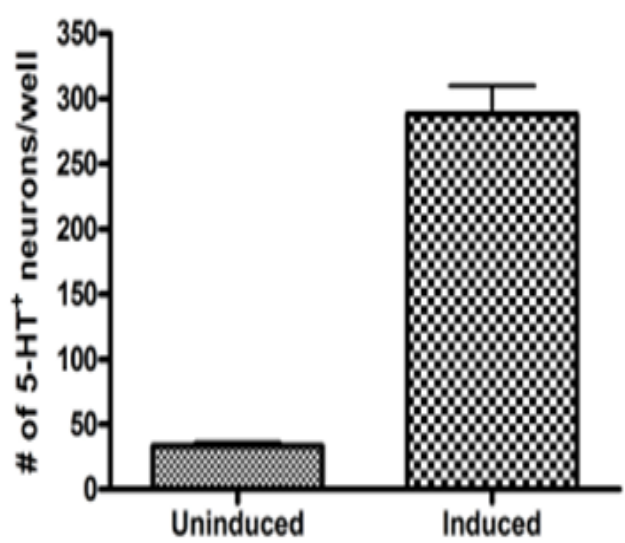

Figure 3 Foxa2 regulates serotonin neuron number. (A) Foxa2 is necessary for the differentiation of serotonin neurons. Abundant serotonin neurons differentiate from control E9.5 hindbrain explants, whereas almost no serotonin neurons are born from foxa2 nullizygous explants. (B) Foxa2 is sufficient to drive the differentiation of serotonin neurons. F4 mouse embryonic stem cells, which express foxa2 inducibly under the control of doxycycline, were differentiated to neurons. Doxycycline induction of foxa2 leads to a greater than eightfold increase in the number of serotonin neurons generated, as compared to uninduced cells. 

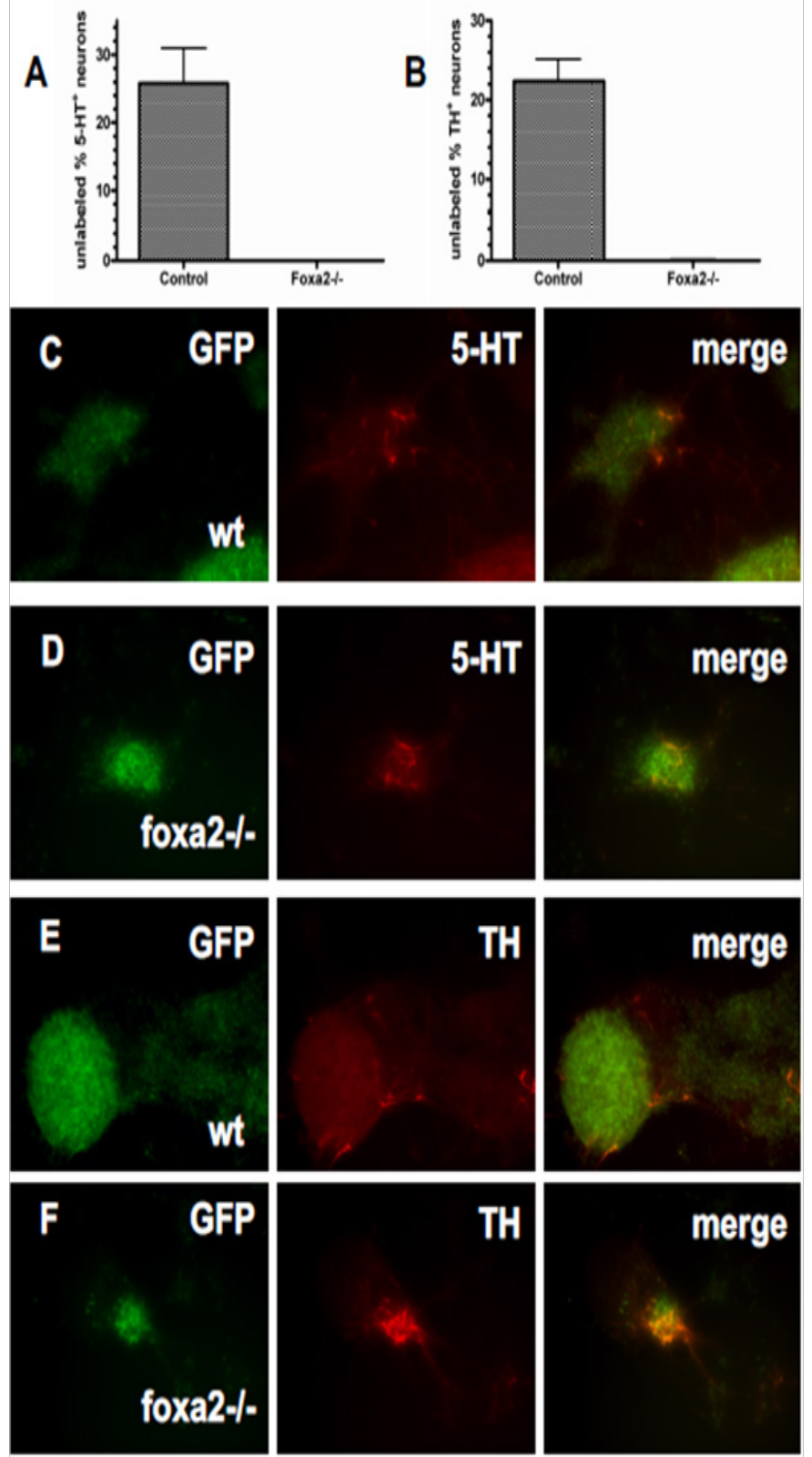

Figure 4 Foxa2 cell-autonomously regulates monoaminergic differentiation in the floor plate. (A) Foxa2 is cell-autonomously required for dopamine neuron differentiation in from mesencephalic floor plate progenitors. E8.5 ventral midbrain progenitors from H2B-GFP transgenic mice were co-cultured with progenitors from either wild-type (C57BL/6) or foxa2-/- embryos. Unlike the wild-type cells, the unlabeled (not GFP-expressing) foxa2-/- midbrain progenitors were unable to differentiate into $\mathrm{TH}$-expressing dopamine neurons in co-culture. Representative images are to the right. (B) Foxa2 is cell-autonomously required for the differentiation of serotonin neurons. E9.5 H2B-GFP hindbrain progenitors were co-cultured with wild-type or foxa2/- hindbrain progenitors. Once again, foxa2-/- progenitors were unable to differentiate into serotonin-immunoreactive neurons, unlike their wild-type counterparts.

\section{Discussion}

It is now widely appreciated that the replacement of dopamine neurons by cells derived from human embryonic stem cells is one of the leading models for the utility of and the excitement surrounding stem cells. Parkinson's disease affects the survival of dopamine neurons and is estimated to afflict 2 million people worldwide making it a prime target for stem cell therapies. It is important to show that stem cell therapies will be broadly applicable to degenerative disease and not only the most prevalent diseases. While the loss of serotonin neurons has not been shown to be widely observed in human diseases. Human genetic variation in serotonin-related genes, such as the serotonin transporter and MAOA has been found to be associated with anxiety, aggression, mental retardation, and mood-related disorders. Abnormal levels of serotonin have been detected in children with autism and ADHD. These findings suggest that perturbation of normal development and early function of serotonin neurons may contribute to abnormal brain function. A better understanding of the biology of the serotonergic system will offer new target genes to identify genetic variants that might contribute to disease.

If the shh and foxa2-expressing floor plate is to undergo neurogenesis, then mechanisms separate from the previously established shh morphogen gradient must be involved in patterning of the floor plate. ${ }^{20}$ An earlier study implicated foxa2 in the generation of serotonin neurons; foxa 2 and phox $2 \mathrm{a}$ were shown to be mutually antagonistic for determining the floor plate boundary and the induction of motoneuron versus serotonin neuron fate. The dopaminergic and serotonergic fates have been shown to be dependent on the expression of shh in the floor plate but, until now, it has not been clear whether foxa2 was cell autonomously required for the differentiation of these important cells. Here, we show, for the first time, that foxa2 is intrinsically required for cells to acquire the midbrain dopaminergic or serotonergic fates. Previously, we have shown that the conditional loss of shh in the hindbrain floor plate leads to neurogenesis, specifically of tyrosine hydroxylase-expressing neurons; conversely, persistent expression or overexpression of shh leads to the suppression of floor plate neurogenesis in the midbrain. ${ }^{28-30}$ Taken together with our previous results, we are left with a model for floor plate differentiation, at least, at the level of the midbrain and hindbrain, in which floor plate expression of sh suppresses neurogenesis of medial floor plate cells while instructing lateral floor plate cells to assume the serotonergic fate. In the absence of shh, the medial floor plate differentiates to dopamine neurons, while the lateral floor plate becomes non-serotonergic and non catecholaminergic. It will be interesting to see if these cell autonomous and non-autonomous pathways are retained in the development of other ventral structures of the CNS. The differentiation of dopamine and serotonin neurons is an important goal in the development of therapies for neurological and neuropsychiatric conditions. These data suggest that protocols to differentiate therapeutically useful, monoaminergic cells will require passage through a floor plate cell-type and this step cannot be replaced by cell culture conditions.

\section{Methods}

\section{Immunohistochemistry and histology}

Timed pregnant CD-1 mice (Charles River Laboratories) were obtained and embryos were manually dissected and fixed in $4 \%$ paraformaldehyde (PFA) in PBS, transferred to $20 \%$ sucrose overnight, and embedded in O.C.T compound (Tissue Tek, Sakra). $12 \mu \mathrm{m}$ sections were cut on a Microm MH 500 OM cryosectioner and processed for immunohistochemistry as previously described. ${ }^{20,21}$ All animal work was carried out in accordance with NIH Animal Studies Protocols as approved by the Animal Care and Use Committee. 


\section{Antibodies}

For immunohistochemistry of mouse embryo sections and fixed cells in vitro, the following antibodies were used: En1/2, Foxa2 and Nkx2.2 (1:10, mouse monoclonal, Developmental Studies Hybridoma Bank, University of Iowa); Foxa2 (1:4,000, rabbit polyclonal, gift of Ariel Ruiz I Altaba); Foxa2 (1:50, goat polyclonal, Santa Cruz Biotechnology); Lmx1b (1:4,000, guinea pig polyclonal, gift of T. Jessell); Lmx1b (1:500, rabbit polyclonal, gift of T. Muller); nestin (1:50, rabbit polyclonal, McKay lab); Nkx2.2 (1:400, guinea pig polyclonal, gift of B. Sosa Pineda); Phox2a (1:800, rabbit polyclonal, gift of J.-F. Brunet); serotonin (1:4,000, rabbit polyclonal, Sigma); $\beta$-tubulin (1:800, mouse monoclonal, Covance); tyrosine hydroxylase (1:400, rabbit and sheep polyclonals, Pel-freez).

\section{Cell culture}

CNS explants were dissected from E9.5 foxa2 -/- embryos and wild-type embryos. Explants were plated directly in $\mathrm{N}_{2}$ medium containing 100ng/ml FGF8 and 500ng/ml shh, as described above. After 4days in culture with mitogens, the explants were grown for four additional days in the absence of exogenous growth factors to promote differentiation. After four days of differentiation, the explants were fixed with $4 \%$ paraformaldehyde.

The mouse embryonic stem cell line, F4, which expresses foxa2 inducibly has been described. ${ }^{21}$ Approximately 50,000 F4 cells were plated per well of a fibronectin-coated, 24-well plate into $\mathrm{N}_{2}$ medium. The cells were differentiated for 28days in $\mathrm{N}_{2}$, in the absence of exogenous growth factors. Foxa2 was induced using doxycycline from days 6-23 of culture (no induction for the first and last five days of the differentiation).

For the co-culture experiments, E8.5 midbrain or E9.5 hindbrain was dissected from H2-GFP (gift of K. Hadjontanakis) C57BL/6, and foxa2-/- embryos, dissociated in trypsin, and plated to fibronectin, as we previously described.$^{20}$ Roughly two embryo equivalents of H2BGFP cells and the cells from a single C57BL/6 or foxa2-/- embryo were plated into a single well of a 24-well plate. The cells were expanded for 3days with shh $(500 \mathrm{ng} / \mathrm{ml})$ and FGF-8 $(100 \mathrm{ng} / \mathrm{ml})$, added each day, and differentiated for 4 days in the absence of growth factors.

\section{Acknowledgements}

None.

\section{Conflict of interest}

The author declares no conflict of interest.

\section{References}

1. Jacobs BL, Azmitia EC. Structure and function of the brain serotonin system. Physiol Rev. 1992;72(1):165-220.

2. Maes M, Meltzer HY. The serotonin hypothesis of major depression. In Bloom FE, Kupfer DJ, editors. Psychopharmacology: the fourth generation of progress. USA: Raven Press; 1995.

3. Griebel G. 5-Hydroxytryptamine-interacting drugs in animal models of anxiety disorders: More than 30 years of research. Pharmacology and Therapeutics. 1995;63(3):319-395.

4. Nelson RJ, Chiavegatto S. Molecular basis of aggression. Trends Neurosci. 2001;24(12):713-719.
5. Rocha BA, Scearce-Levie K, Lucas JJ, et al. Increased vulnerability to cocaine in mice lacking the serotonin-1B receptor. Nature. 1998;393(6681):175-178.

6. Winstanley CA, Theobald DE, Dalley JW, et al. Interactions between serotonin and dopamine in the control of impulsive choice in rats: therapeutic implications for impulse control disorders. Neuropsychopharamacology. 2004;30(4):669-682.

7. Meltzer HY. The role of serotonin in antipsychotic drug action. Neuropsychopharmacology. 1999;21(2 Suppl):106S-115S.

8. Fineberg NA, Brown A, Reghunandanan S, et al. Evidence-based pharmacotherapy of obsessive-compulsive disorder. Int J Neuropsycholopharmacol. 2005;15(8):1173-1191.

9. Mitchell JE, de Zwaan M, Roerig JL. Drug therapy for patients with eating disorders. Curr Drug Targets CNS Neurol Disord. 2003;2(1):17-29.

10. Paterson DS, Trachtenberg FL, Thompson EG, et al. Multiple serotonergic brainstem abnormalities in sudden infant death syndrome. JAMA. 2006;296(17):2124-2132.

11. Chugani DC, Muzik O, Behen M, et al. Developmental changes in brain serotonin synthesis capacity in autistic and non autistic children. Ann Neurol. 1999;45(3):287-295.

12. Gross C, Zhuang X, Stark K, et al. Serotonin ${ }_{1 \mathrm{~A}}$ receptor acts during development to establish normal anxiety-like behaviour in the adult. $\mathrm{Na}$ ture. 2002;416(6879):396-400.

13. Hendricks TJ, Fyodorov DV, Wegman LJ, et al. Pet-1 ETS gene plays a critical role in 5-HT neuron development and is required for normal anxiety-like and aggressive behavior. Neuron. 2003;37(2):233-247.

14. Sundstrom E, Kölare S, Souverbie F, et al. Neurochemical differentiation of human bulbospinal monoaminergic neurons during the first trimester. Brain Res Dev Brain Res. 1993;75(1):1-12.

15. Goridis C, Rohrer H. pecification of catecholaminergic and serotonergic neurons. Nat Rev Neurosci. 2002;3(7):531-541.

16. Hynes M, Rosenthal A. Specification of dopaminergic and serotonergic neurons in the vertebrate CNS. Curr Opin Neurobiol. 1999;9(1):26-36.

17. Ye W, Shimamura K, Rubenstein JL, et al. FGF and Shh signals control dopaminergic and serotonergic cell fate in the anterior neural plate. Cell. 1998;93(5):755-766.

18. Kim JH, Auerbach JM, Rodríguez-Gómez JA, et al. Dopamine neurons derived from embryonic stem cells function in an animal model of Parkinson's disease. Nature. 2002;418(6893):50-56.

19. Mann JJ. The medical management of depression. $N$ Engl J Med. 2005;353(17):1819-1834

20. Wurst W, Bally-Cuif L. Neural plate patterning: upstream and downstream of the isthmic organizer. Nat Rev Neurosci. 2001;2(2):99-108.

21. Kittappa R, Chang WW, Awatramani R, et al. Spontaneous degeneration of dopamine neurons in old age. PLoS Biology. 2007;5(12):e325.

22. Briscoe J, Pierani A, Jessell TM, et al. A homeodomain protein code specifies progenitor cell identity and neuronal fate in the ventral neural tube. Cell. 2000;101(4):435-445.

23. Matise MP, Epstein DJ, Park HL, et al. Gli2 is required for induction of floor plate and adjacent cells, but not most ventral neurons in the mouse central nervous system. Development. 1998;125(15):2759-70.

24. Craven SE, Lim KC, Ye W, et al. Gata2 specifies serotonergic neurons downstream of sonic hedgehog. Development. 2004;131(5):1165-1173. 
25. Pattyn A, Vallstedt A, Dias JM, et al. Coordinated temporal and spatial control of motor neuron and serotonergic neuron generation from a common pool of CNS progenitors. Genes Dev. 2003;17(6):729-37.

26. Briscoe J, Sussel L, Serup P, et al. Homeobox gene Nkx2.2 and specification of neuronal identity by graded Sonic hedgehog signalling. Nature. 1999;398(6728):622-627.

27. Simon $\mathrm{HH}$, Saueressig H, Wurst W, et al. Fate of midbrain dopaminergic neurons controlled by the engrailed genes. JNeurosci. 2001;21(9):31263134.
28. Lee SH, Nadya L, Lorenz S, et al. Efficient generation of midbrain and hindbrain neurons from mouse embryonic stem cells. Nat Biotechnol. 2000;18:675-679.

29. Jacob J, Ferri AL, Milton C, et al. Transcriptional repression coordinates the temporal switch from motor to serotonergic neurogenesis. $\mathrm{Nat} \mathrm{Neu}$ rosci. 2007;10(11):1433-1439.

30. Lu J, Xuefei Zhong, Huisheng Liu, et al. Generation of serotonin neurons from human pluripotent stem cells. Nat Biotechnol. 2016;34:89-94. 